\title{
Value of systematic biopsy added to target biopsy for detecting significant cancer in men with Prostate Imaging and Reporting and Data System 5
}

\author{
Taein An, Byung Kwan Park \\ Department of Radiology, Samsung Medical Center, Sungkyunkwan University School of Medicine, Seoul, Korea
}

Received: July 2, 2020

Revised: July 23, 2020

Accepted: July 24, 2020

Corresponding author:

Byung Kwan Park

Department of Radiology,

Samsung Medical Center,

Sungkyunkwan University

School of Medicine, 81 Irwon-ro,

Gangnam-gu, Seoul 06351, Korea

Tel: +82-2-3410-6457

E-mail: rapark@skku.edu

\begin{abstract}
Purpose: To assess the value of systematic biopsy added to target biopsy for detecting significant cancer in men with Prostate Imaging and Reporting and Data System 5 (PIRADS 5).

Methods: Between March 2014 and November 2018, 186 men had a PI-RADS 5 categorized as an index lesion on magnetic resonance imaging prior to transrectal ultrasound (TRUS)-guided biopsy. Of these patients, 135 (group I) underwent target biopsy alone because of good depiction. The remaining 51 (group II) underwent target and systematic biopsies because of poor depiction. Significant cancer detection rates (CDRs) were compared between the groups. Which type of biopsies contributed to detecting significant cancer was evaluated in the group II.

Results: Significant CDRs of the target biopsy were $67.4 \%$ (91/135) in the group I and $47.1 \%(24 / 51)$ in the group II $(P=0.0173)$. However, when systematic biopsy was added to target biopsy, the significant CDR of the group II increased to $52.9 \%(27 / 51)(P=0.0900)$. Of the 27 significant cancers missed by target biopsy in the group II, three were detected by systematic biopsy alone. Moreover, systematic biopsy detected higher Gleason scores in two cases than target biopsy.

Conclusion: Systematic biopsy contributes to detecting additional significant cancers in men with PI-RADS 5 partially visible on TRUS.
\end{abstract}

Keywords: Biopsy; Magnetic resonance imaging; Male; Prostate; Ultrasonography
This is an Open Access article distributed under the terms of the Creative Commons Attribution Non-Commercial License (https:// creativecommons.org/licenses/ by-nc/4.0/).

\section{INTRODUCTION}

Recently, Prostate Imaging and Reporting and Data System version 2 (PI-RADSv2) has been introduced to the clinical practice [1-3]. PI-RADSv2 standardizes the interpretation and reporting of multi-parametric magnetic resonance imaging (MRI) and subsequently enhance communication between radiologists and urologists. But also, it helps to stratify the risk of prostate cancer [1-3]. Many investigators have reported that PI-RADSv2 contributes to detecting significant prostate cancer [4-9]. 
Prostate Imaging and Reporting and Data System (PIRADS) 5 is very likely to have significant cancer and thus biopsy is strongly recommended [1-3]. This lesion is larger or more aggressive than that of the other categories so that it is clearly seen on MRI. Frequently, target biopsy alone is enough to detect significant cancer [1-3]. However, the likelihood of significant cancer is not $0 \%$ even though the other prostate is categorized as PI-RADS 1, in which the multiparametric magnetic resonance (MR) features are unremarkable. Moreover, PI-RADS 5 does not guarantee significant cancer detection $100 \%$. Therefore, it is still unclear if addition of systematic biopsy to target biopsy is necessary in detecting significant cancer. Our hypothesis was that systematic biopsy provides additional value to target biopsy for detection of significant cancer in men with PI-RADS 5.

The purpose of our study was to assess the value of systematic biopsy added to target biopsy for detecting significant cancer in men with PI-RADS 5.

\section{METHODS}

\section{Patient selection}

Between March 2014 and November 2018, 850 men underwent prostate biopsy, which was performed by a single ra- diologist with 16-year experience of genitourinary intervention (Fig. 1). Among these patients, 664 were excluded due to PI-RADS 1-4 lesions ( $\mathrm{n}=621)$, low $(<2.5 \mathrm{ng} / \mathrm{mL}$ ) prostate specific antigen (PSA; $n=20)$, previous treatments $(n=14)$, no pre-biopsy MRI $(n=5)$, transperineal biopsy $(n=2)$, poor quality MRI images $(n=1)$, and metastatic prostate cancer $(n=1)$.

The remaining 186 men (median, 68 years; range, 35 to 86) met for the following inclusion criteria: high ( $\geq 2.5 \mathrm{ng} / \mathrm{mL}$ ) PSA, PI-RADS 5 lesion, pre-biopsy MRI, and transrectal ultrasound (TRUS)-guided biopsy. Their PSA, prostate volume, PSA density, MRI-biopsy interval, and tumor size ranged 2.54 to $2,564.38 \mathrm{ng} / \mathrm{mL}$ (median, 7.22), 13 to $203 \mathrm{~mL}$ (median, 35), 0.05 to $68.6 \mathrm{ng} / \mathrm{mL}^{2}$ (median, 0.20 ), 0 to 397 days (median, 31), and 15 to $57 \mathrm{~mm}$ (median, 19), respectively. These patients were divided into group I $(n=135)$ and group II $(n=51)$. The group I had 135 PI-RADS 5 lesions which were completely visible on TRUS. The group II had 51 PI-RADS 5 lesions which were partially visible on TRUS. Patient demographics in each group were summarized in the Table 1.

\section{Prostate MRI and biopsy}

Prior to prostate biopsy, multi-parametric MRI was scanned to obtain the following sequences: T2-weighted, diffusion-weighted, and dynamic contrast-enhanced axial MR im-

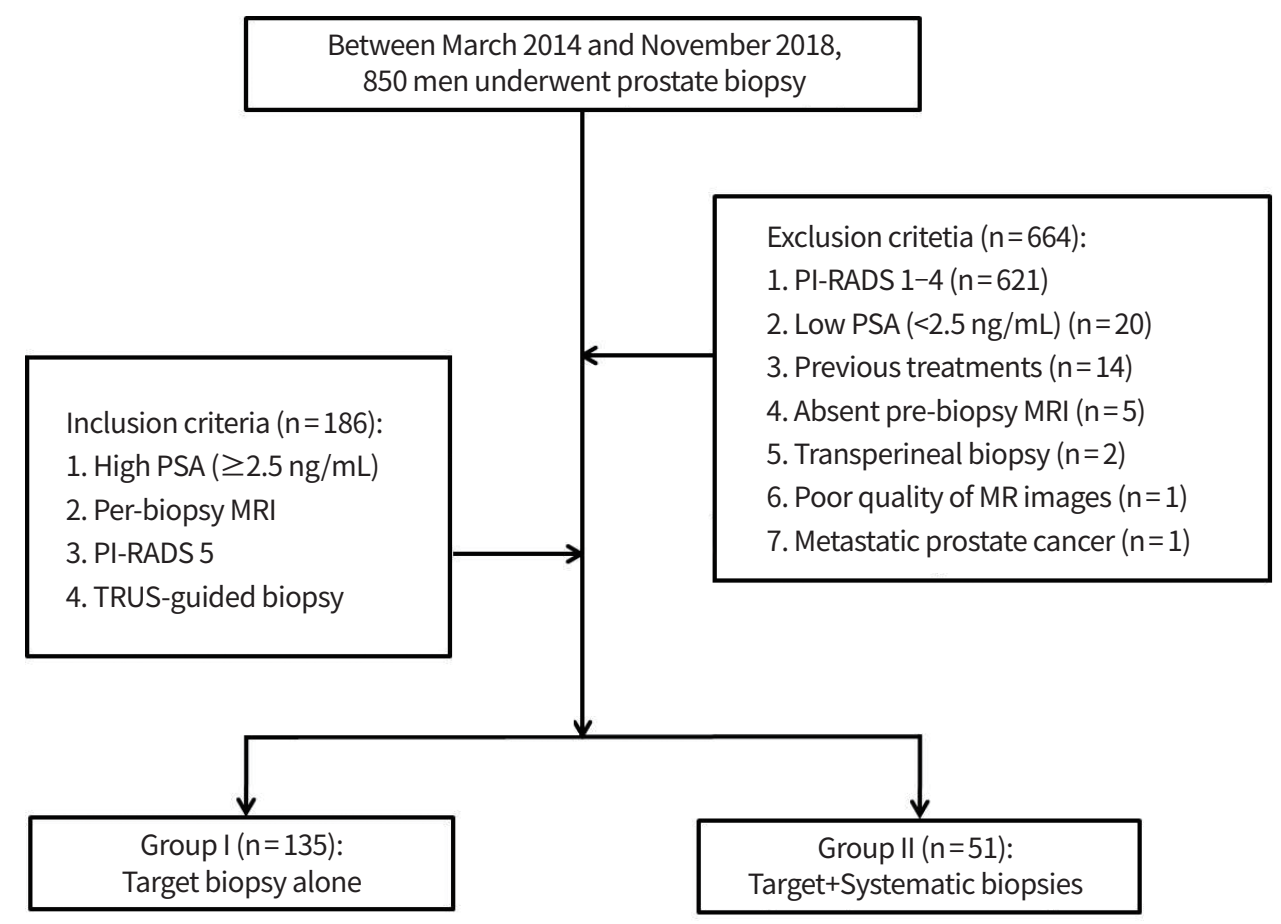

Fig. 1. Flow diagram of study population. PI-RADS, Prostate Imaging Reporting and Data System; PSA, prostate specific antigen; MRI, magnetic resonance imaging; MR, magnetic resonance; TRUS, transrectal ultrasound. 
ages. A single radiologist interpreted MR images and then performed target and/or systematic biopsy under TRUS guidance. He was already familiar with the following TRUS techniques and imaging features: (1) prostate was not compressed for lesion detection by a probe, (2) a TRUS lesion was located higher than an MRI lesion, and (3) a significant cancer appeared hypoechoic in peripheral zone and hyperechoic in transition zone. Group I underwent target biopsy alone because their PI-RADS 5 lesions were completely visible on TRUS. In the group II, systematic biopsy was added to target biopsy because their PI-RADS 5 lesions were partially visible on TRUS (Fig. 2). This biopsy was performed to systematically sample cores where PI-RADS 4 was not located.

Table 1. Patient demographics

\begin{tabular}{lccc}
\hline & \multicolumn{2}{c}{ PI-RADS 5 cases $(n=186)$} & \\
\cline { 2 - 3 } Demographic & Group I $(n=135)$ & Group II $(n=51)$ & \\
\hline Age $(\mathrm{yr})$ & $68(35-87)$ & $69(51-86)$ & 0.7187 \\
PSA $(\mathrm{ng} / \mathrm{mL})$ & $7.3(2.5-2,783.6)$ & $7.2(2.9-52.5)$ & 0.3935 \\
$\begin{array}{l}\text { Prostate volume } \\
\text { (mL) }\end{array}$ & $33(13-203)$ & $42(19-304)$ & 0.0263 \\
PSAD $\left(\mathrm{ng} / \mathrm{mL}^{2}\right)$ & $0.23(0.05-108.73)$ & $0.18(0.05-2.78)$ & 0.0141 \\
MRI-biopsy interval & $27(0-397)$ & $39(0-308)$ & 0.0049 \\
$\quad$ (day) & & & \\
\hline
\end{tabular}

Values are presented as median (range).

PI-RADS, Prostate Imaging Reporting and Data System; PSA, prostate specific antigen; PSAD, prostate specific antigen density; MRI, magnetic resonance imaging.

\section{Data analysis}

MRI images before 2015 were reloaded into Picture Archiving and Communication System (PACS) to review and to categorize prostate lesions with PI-RADSv2. Since 2015, when PIRADSv2 was released, the original PI-RADS scores categorized by a single radiologist prior to biopsy were used for analysis.

Overall cancer detection rate (CDR) was calculated by the number of cancer cases divided by the number of biopsy cases in each group or each biopsy. Significant cancer was defined as one with Gleason score (GS) $\geq 7$. Significant CDR was calculated by the number of GS $\geq 7$ cases divided by the number of biopsy cases in each group or each biopsy. Overall and significant CDRs were compared between the groups or between the biopsies.

Positive core rate (PCR) was calculated by the number of cancer cores divided by the number of biopsy cores. PCR was compared between the groups or between the target biopsies. PCR was also compared between the target and systematic biopsies in the group II.

The size of peripheral PI-RADS 5 was measured at diffusion-weighted axial images and that of transition PI-RADS 5 was measured at T2-weighted axial MR images. When PIRADS 5 involved both, the tumor size was measured at diffusion-weighted or T2-weighted MR images depending on which zone it was located more dominantly. The median number of total or cancer cores were compared between the groups and between the biopsies.

The medians of the greatest GS (top GS) were compared
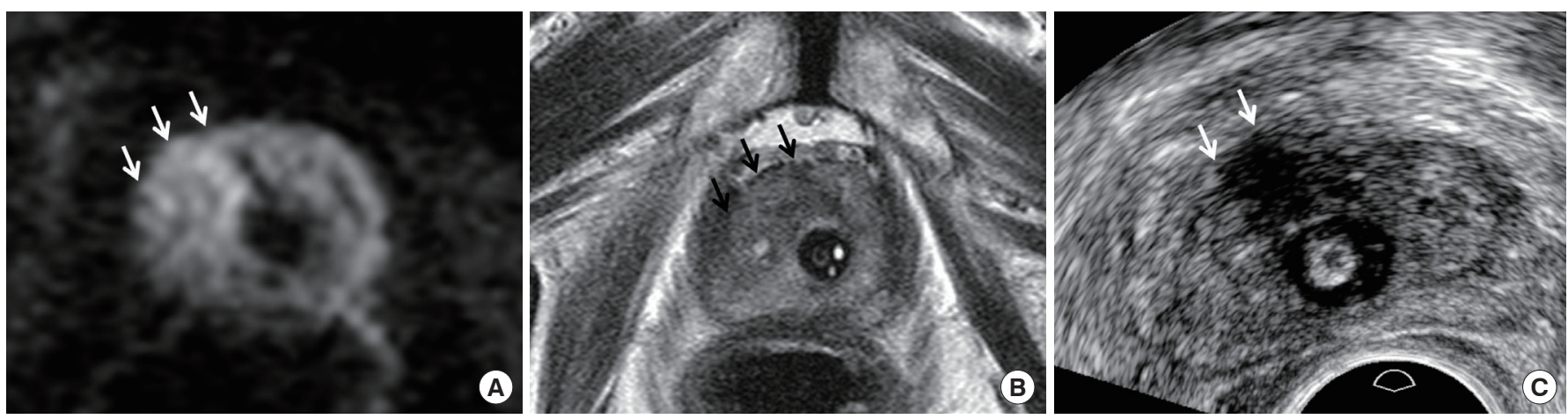

Fig. 2. An 86-year-old man with a Prostate Imaging Reporting and Data System (PI-RADS) 5 cancer partially visible on transrectal ultrasound. (A) Diffusion-weighted axial magnetic resonance (MR) image shows a hyperintense peripheral lesion (white arrows) in the right apex. The tumor margin is not clearly demarcated and the size is measured $18 \mathrm{~mm}$. (B) T2-weighted axial MR image shows a hypointense peripheral lesion (black arrows) in the right apex. Diffusion-weighted or T2-weighted MR findings are consistent with the category of PI-RADS 5. His prostate specific antigen was $51.12 \mathrm{ng} / \mathrm{mL}$. (C) Transrectal ultrasound-guided axial image shows a hypoechoic peripheral lesion (white arrows) in the right apex. However, it is not well correlated with one on magnetic resonance imaging in terms of lesion size or shape because of partial lesion visibility. Target biopsy confirmed Gleason score $6(3+3)$ adenocarcinoma, but systematic biopsy did Gleason score $7(3+4)$ in the left apex. 
between the groups. Percentage length of top GS core was defined as the proportion of cancer length compared to the entire length of a biopsy core. The percentage length was also compared between the groups when targeting biopsy was performed.

Which type of biopsies contributed to detecting insignificant or significant cancer was evaluated in the group II. GSS were compared between target and systematic biopsies to determine how many systematic biopsies achieved higher GS than target biopsy.

\section{Statistical analysis}

Mann-Whitney test was used to compare patient age, PSA, prostate volume, PSA density, MRI-biopsy interval, tumor size, top GS, and percentage length of significant cancer core between the groups. Fisher's exact test was used to compare CDRs and PCRs between the groups or the biopsies. Odds ratio (OR) was obtained to quantify the strength of the association between the biopsies in targeting PI-RADS 5 completely or partially visible on TRUS. The approximation of Woolf was used to calculate $95 \%$ confidence interval. Wilcoxon matchedpairs signed-ranks test was used to compare cores between target and systematic biopsies in the group II. Commercially available software (PASW Statistics, version 20.0; IBM Co., Armonk, NY, USA) was used for statistical analysis. A two-side $P$-value of less than 0.05 was considered statistically significant.

\section{Ethical statement}

This retrospective study was approved by Institutional Re-

Table 2. Comparison of target biopsy outcomes according to the lesion visibility on TRUS

\begin{tabular}{lcccc}
\hline & \multicolumn{2}{c}{$\begin{array}{c}\text { TRUS-guided target } \\
\text { biopsy }(\mathrm{n}=186)\end{array}$} & $\begin{array}{c}\text { OR } \\
\text { Biopsy outcome }\end{array}$ & P-value \\
\cline { 2 - 3 } & $\begin{array}{c}\text { Group I } \\
(\mathrm{n}=135)\end{array}$ & $\begin{array}{c}\text { Graoup II } \\
(\mathrm{n}=51)\end{array}$ & & \\
\cline { 1 - 4 } Overall CDR (\%) & 82.2 & 60.8 & 2.984 & 0.0035 \\
& $(111 / 135)$ & $(31 / 51)$ & $(1.460-$ & \\
& & & $6.098)$ & \\
Significant CDR & 67.4 & 47.1 & 2.327 & 0.0173 \\
(\%) & $(91 / 135)$ & $(24 / 51)$ & $(1.206-$ & \\
& & & $4.490)$ & \\
PCR (\%) & 71.2 & 48.1 & 2.665 & $<0.0001$ \\
& $(532 / 747)$ & $(143 / 297)$ & $(2.019-$ & \\
& & & $3.517)$ & \\
& & & &
\end{tabular}

TRUS, transrectal ultrasound; OR, odds ratio; $\mathrm{Cl}$, confidence interval; $\mathrm{CDR}$, cancer detection rate; $\mathrm{PCR}$, positive core rate. view Board of Samsung Medical Center (IRB number: 201901-068) and informed consent was waived.

\section{RESULTS}

Group I had smaller prostate volume $(P=0.0263)$, higher PSA density $(P=0.0141)$, and shorter MRI-biopsy interval $(P=0.0049)$ than group II (Table 1). There was no difference between the groups in terms of age ( $P=0.7187)$ and PSA $(P=0.3935)$.

The overall CDRs of the target biopsy were $82.2(111 / 135)$ in the group I and $60.8(31 / 51)$ in the group II $(P=0.0035$ and $\mathrm{OR}=2.984$ ) (Table 2). The significant CDRs of the target biopsy were $67.4(91 / 135)$ in the group I and $47.1(24 / 51)$ in the group II $(P=0.0173$ and $O R=2.327)$. The PCRs of the target biopsy were $71.2(532 / 747)$ in the group I and $48.1(143 / 297)$ in the group II $(\mathrm{P}<0.0001$ and $\mathrm{OR}=2.665)$.

However, when the outcomes of systematic biopsy were added to those of target biopsy in the group II, there was no difference between group I and II in terms of overall CDR $(P=0.0734$ and $O R=2.030)$ and significant $C D R(P=0.0900$ and $O R=1.798)$. Still, the $P C R$ of the group I was much higher than that of the group II $(P<0.0001$ and $O R=5.541)$ (Table 3).

The median tumor sizes were $20 \mathrm{~mm}$ (range, 15 to 57 ) in the group I and $18 \mathrm{~mm}$ (range, 15 to 50$)$ in the group II ( $P=0.1268)$ (Table 4). Target biopsy yielded a greater number of median cancer cores in the group I than group II $(P=0.0303)$ although the number of target biopsy cores was smaller in the former than the latter $(P=0.0345)$. There was no difference between group I and II in terms of top $G S(P=0.0993)$ and percentage

Table 3. Comparison of biopsy outcomes between group I (target) and II (target+systematic)

\begin{tabular}{|c|c|c|c|c|}
\hline \multirow{2}{*}{ Biopsy outcome } & \multicolumn{2}{|c|}{$\begin{array}{l}\text { TRUS-guided biopsy } \\
\qquad(n=186)\end{array}$} & \multirow{2}{*}{$\begin{array}{c}\text { OR } \\
(95 \% \mathrm{Cl})\end{array}$} & \multirow{2}{*}{ P-value } \\
\hline & $\begin{array}{l}\text { Group I } \\
(n=135)\end{array}$ & $\begin{array}{l}\text { Group II } \\
(n=51)\end{array}$ & & \\
\hline Overall CDR (\%) & $\begin{array}{c}82.2 \\
(111 / 135)\end{array}$ & $\begin{array}{c}68.6 \\
(35 / 51)\end{array}$ & $\begin{array}{c}2.03 \\
(0.9745- \\
4.228)\end{array}$ & 0.0734 \\
\hline Significant CDR (\%) & $\begin{array}{c}67.4 \\
(91 / 135)\end{array}$ & $\begin{array}{c}52.9 \\
(27 / 51)\end{array}$ & $\begin{array}{c}1.798 \\
(0.9329- \\
3.463)\end{array}$ & 0.0900 \\
\hline PCR (\%) & $\begin{array}{c}71.2 \\
(532 / 747)\end{array}$ & $\begin{array}{c}30.9 \\
(188 / 609)\end{array}$ & $\begin{array}{c}5.541 \\
(4.386- \\
7.001)\end{array}$ & $<0.0001$ \\
\hline
\end{tabular}

TRUS, transrectal ultrasound; OR, odds ratio; $\mathrm{Cl}$, confidence interval; $\mathrm{CDR}$, cancer detection rate; $\mathrm{PCR}$, positive core rate. 
Table 4. Comparison of group I and II in terms of lesion size and biopsy features

\begin{tabular}{lccc}
\hline \multirow{2}{*}{$\begin{array}{c}\text { Lesion size and } \\
\text { biopsy features }\end{array}$} & $\begin{array}{c}\text { Group I } \\
(n=136)\end{array}$ & $\begin{array}{c}\text { Group II } \\
(n=128)\end{array}$ & P-value \\
\cline { 2 - 3 } & $20(15-57)$ & $18(15-50)$ & 0.1268 \\
\hline $\begin{array}{l}\text { Tumor size }(\mathrm{mm}) \\
\text { No. of target cores }\end{array}$ & $5(2-12)$ & $6(1-10)$ & 0.0345 \\
$\begin{array}{l}\text { No. of target cancer } \\
\text { cores }\end{array}$ & $4(0-12)$ & $3(0-8)$ & 0.0303 \\
$\begin{array}{l}\text { Top GS } \\
\text { a) }\end{array}$ & $7(6-9)$ & $7(6-8)$ & 0.0993 \\
$\begin{array}{c}\text { \% length of top GS } \\
\text { core }^{\text {a) }}\end{array}$ & $70(5-100)$ & $70(5-95)$ & 0.5650 \\
\hline
\end{tabular}

Values are presented as median (range).

PI-RADS, Prostate Imaging Reporting and Data System; GS, Gleason score.

a) Top GS and \% length of top GS core obtained from target biopsy were compared between the groups.

length of top GS core ( $P=0.5650)$ (Fig. 2).

In the group II, overall CDRs of target and systematic biopsies were $60.8(31 / 51)$ and $47.1(24 / 51)(P=0.2332$ and $O R=1.744)$ (Table 5). However, the significant CDRs of theses biopsies were $47.1(24 / 51)$ and $23.5(12 / 51)(P=0.0220$ and $\mathrm{OR}=2.889)$. The PCRs of these biopsies were 48.1 (143/297) and 14.4 $(45 / 312)(P<0.0001$ and $O R=5.510)$. The number of cores were 297 (median, 6; range, 1 to 10) in the target biopsy and 312 (median, 6; range, 3 to 12 ) in the systematic biopsy ( $P>0.9999)$. Of the 31 cancer cases, the number of cancer cores were 143 (median, 5; range, 1 to 8 ) in the target biopsy and 40 (median, 1; range, 0 to 5$)$ in the systematic biopsy $(P<0.0001)$.

Of the 27 significant cancers in the group II, 15 were detected by target biopsy, nine by target and systematic biopsies, and three by systematic biopsy. Of the three significant cancers detected by systematic biopsy alone, two and one were diagnosed as GS 6 tumors and a benign tissue by target biopsy, respectively (Fig. 2). One GS 7 (3+4) tumor was diagnosed by target biopsy, whereas it was confirmed as a GS $8(4+4)$ tumor by systematic biopsy.

\section{DISCUSSION}

MRI-TRUS fusion or in-bore MRI-guided biopsy targets PI-RADS 5 because significant cancer is very likely to be present [1-3]. When the other prostate tissue except PI-RADS 5 is PI-RADS 1 or 2 , additional target biopsy is not so effective because of low CDRs. Several investigations have reported that the CDRs of bi-
Table 5. Comparison of target and systematic biopsies in the group II

\begin{tabular}{|c|c|c|c|c|}
\hline \multirow{2}{*}{$\begin{array}{l}\text { Biopsy } \\
\text { outcomes }\end{array}$} & \multicolumn{2}{|c|}{ Group II $(n=51)$} & \multirow[b]{2}{*}{ OR $(95 \% \mathrm{Cl})$} & \multirow[b]{2}{*}{ P-value } \\
\hline & $\begin{array}{l}\text { Target } \\
\text { biopsy }\end{array}$ & $\begin{array}{l}\text { Systemat- } \\
\text { ic biopsy }\end{array}$ & & \\
\hline Overall CDR (\%) & $\begin{array}{c}60.8 \\
(31 / 51)\end{array}$ & $\begin{array}{c}47.1 \\
(24 / 51)\end{array}$ & $\begin{array}{c}1.744 \\
(0.7941- \\
3.829)\end{array}$ & 0.2332 \\
\hline Significant CDR (\%) & $\begin{array}{c}47.1 \\
(24 / 51)\end{array}$ & $\begin{array}{c}23.5 \\
(12 / 51)\end{array}$ & $\begin{array}{c}2.889 \\
(1.236- \\
6.754)\end{array}$ & 0.0220 \\
\hline PCR (\%) & $\begin{array}{c}48.1 \\
(143 / 297)\end{array}$ & $\begin{array}{c}14.4 \\
(45 / 312)\end{array}$ & $\begin{array}{c}5.51 \\
(3.732- \\
8.133)\end{array}$ & $<0.0001$ \\
\hline
\end{tabular}

OR, odds ratio; $\mathrm{Cl}$, confidence interval; $\mathrm{CDR}$, cancer detection rate; PCR, positive core rate.

opsy in PI-RADS 1 or 2 range from $14 \%$ to $25 \%[4,6,8,10]$. PIRADSV2 definitely recommends a biopsy in the PI-RADS 5 cases but does not show how to do it. Still, urologists or radiologists do not know that systematic biopsy helps additional detection of significant cancer if it is combined with target biopsy. Simply increasing the number of cores also run the higher risk of biopsy-related complications such as pain, urinary retention, bleeding, or infection [11-14].

If only a few cores detect significant cancer, target biopsy alone is ideal for correct diagnosis and rare complications. The significant CDR of the target biopsy in the group I is not inferior to those in previously reported papers using MRITRUS fusion or in-bore MRI-guided biopsy. They have showed that their CDRs ranged from $62 \%$ to $85 \%$ in PI-RADS 5 [8, $10,15,16]$. However, PI-RADS 1 or 2 does not have significant cancer $100 \%$ even though this category is very unlikely or unlikely to have it [1-3]. Moreover, because various kinds of inflammation can mimic prostate cancer, significant cancer cannot be detected if target biopsy alone is performed [17-19].

Our study demonstrated that systematic biopsy detected additional significant cancers in the PI-RADS 1 or 2 , although target biopsy did not find significant cancer in the PI-RADS 5 . When PI-RADS 5 was completely visible on TRUS (group I), the CDR of the target biopsy was better than that of the target biopsy in PI-RADS 5 which was partially visible on TRUS (group II). Accordingly, lesion visibility on TRUS may influence the detection of significant cancer. These findings suggest that systematic biopsy is necessary if PI-RADS 5 does not appear completely visible on TRUS.

Our study showed another evidence that systematic biop- 


\section{PRECISION AND FUTURE MIEDICINE}

Systematic biopsy of PI-RADS 5

sy is necessary. The overall and significant CDRs of the target biopsy in the group I were statistically different from those of the target biopsy in the group II. However, after systematic biopsy was added to target biopsy in the group II, the overall and significant CDRs were elevated so that group II were not different from group I in terms of CDR. The overall and significant CDRs in the group II became higher $6 \%$ to $9 \%$ by systematic biopsy added to target biopsy. During the in-bore MRI-guidance, target biopsy is preferred to use, whereas systematic biopsy is hard to perform because it is considered time-consuming and inefficient procedures for detecting significant cancer in the PI-RADS 5 cases. From this point of view, in-bore MRI-guided biopsy might be inferior to MRITRUS fusion biopsy using both target and systematic biopsy. Venderink et al. [16] have reported that there was no difference between the two types of biopsy techniques. A systematic review also shows that in-bore MRI-guided biopsy is not different from MRI-TRUS fusion in terms of overall and significant CDRs [20]. However, these studies did not compare inbore MRI-guided target biopsy and MRI-TRUS fusion target and systematic biopsies.

There are other reasons for necessity of systematic biopsy in the PI-RADS 5 cases. First, prostate cancer is more likely to be multifocal in the PI-RADS 5 compared to the other categories. Multifocal significant cancers are frequently encountered in the post-prostatectomy specimens [21-23]. Systematic biopsy may detect so small significant cancers which are hard to find on MRI. Second, it is not uncommon that a small lesion may have higher GS than a large lesion. Our study showed that the GSs of two cases were upgraded in the systematic biopsy than in the target biopsy. Third, it should be kept in mind that PI-RADS 5 may not be targeted. Even if the TRUS guideline of target biopsy is placed in the lesion center, the biopsy needle may be shot somewhat off the center. Therefore, one or two biopsy cores may be insufficient to reveal the small component of GS 4 or 5 . Our study showed that the median number of target cores were 5 in complete visible PI-RADS 5 on TRUS. Still, the optimal number of target cores is unclear and further investigation is necessary.

Our study had some limitations. First, the CDR of target biopsy in the group I can be underestimated because systematic biopsy was not performed. Even though PI-RADS 5 is completely visible, the necessity of systematic biopsy should be evaluated. Second, what systematic protocol is optimal for PI-RADS 5 is still unknown. It requires maximizing CDR and minimizing complications. Third, what number of target cores is ideal is still unknown. If the component of GS 4 or 5 is small and eccentric, significant cancer cannot be detected with only one or two target cores. Fourth, transition PI-RADS 5 was not evaluated because of a relatively small number of cases. Last, the design of our study was retrospective.

In conclusion, TRUS-guided target biopsy provides higher overall and significant CDRs in the completely visible PIRADS 5 than partially visible PI-RADS 5. However, systematic biopsy has a potential to detect additional significant cancers in the other prostate when target biopsy does not sample significant cancer from the partially visible PI-RADS 5 on TRUS. Therefore, systematic biopsy as well as target biopsy is recommended in the PI-RADS 5 cases.

\section{CONFLICTS OF INTEREST}

No potential conflict of interest relevant to this article was reported.

\section{ORCID}

Taein An https://orcid.org/0000-0002-0569-6022

Byung Kwan Park https://orcid.org/0000-0002-4114-8859

\section{AUTHOR CONTRIBUTIONS}

Conception or design: BKP.

Acquisition, analysis, or interpretation of data: TA, BKP.

Drafting the work or revising: TA, BKP.

Final approval of the manuscript: TA, BKP.

\section{REFERENCES}

1. Weinreb JC, Barentsz JO, Choyke PL, Cornud F, Haider MA, Macura KJ, et al. PI-RADS prostate imaging: reporting and data system: 2015, version 2. Eur Urol 2016;69:16-40.

2. Barentsz JO, Weinreb JC, Verma S, Thoeny HC, Tempany CM, Shtern F, et al. Synopsis of the PI-RADS v2 guidelines for multiparametric prostate magnetic resonance imaging and recommendations for use. Eur Urol 2016;69:41-9.

3. Purysko AS, Rosenkrantz AB, Barentsz JO, Weinreb JC, Macura KJ. PI-RADS version 2: a pictorial update. Radiographics 2016;36:1354-72.

4. Greer MD, Shih JH, Lay N, Barrett T, Kayat Bittencourt L, Borofsky $S$, et al. Validation of the dominant sequence paradigm and role of dynamic contrast-enhanced imaging in PI-RADS version 2. Radiology 2017;285:859-69.

5. Hofbauer SL, Maxeiner A, Kittner B, Heckmann R, Rei- 


\section{PRECISION AND FUTURE MIEDICINE}

Taein An, et al.

mann $\mathrm{M}$, Wiemer L, et al. Validation of prostate imaging reporting and data system version 2 for the detection of prostate cancer. J Urol 2018;200:767-73.

6. Mehralivand S, Bednarova S, Shih JH, Mertan FV, Gaur S, Merino MJ, et al. Prospective evaluation of PI-RADS ${ }^{T M}$ version 2 using the international society of urological pathology prostate cancer grade group system. J Urol 2017; 198:583-90.

7. Park SY, Jung DC, Oh YT, Cho NH, Choi YD, Rha KH, et al. Prostate cancer: PI-RADS version 2 helps preoperatively predict clinically significant cancers. Radiology 2016;280:108-16.

8. Tan N, Lin WC, Khoshnoodi P, Asvadi NH, Yoshida J, Margolis DJ, et al. In-bore 3-T MR-guided transrectal targeted prostate biopsy: prostate imaging reporting and data system version 2-based diagnostic performance for detection of prostate cancer. Radiology 2017;283:130-9.

9. Thai JN, Narayanan HA, George AK, Siddiqui MM, Shah P, Mertan FV, et al. Validation of PI-RADS version 2 in transition zone lesions for the detection of prostate cancer. Radiology 2018;288:485-91.

10. Cash H, Maxeiner A, Stephan C, Fischer T, Durmus T, Holzmann J, et al. The detection of significant prostate cancer is correlated with the Prostate Imaging Reporting and Data System (PI-RADS) in MRI/transrectal ultrasound fusion biopsy. World J Urol 2016;34:525-32.

11. Nam RK, Saskin R, Lee Y, Liu Y, Law C, Klotz LH, et al. Increasing hospital admission rates for urological complications after transrectal ultrasound guided prostate biopsy. J Urol 2010;183:963-8.

12. Loeb S, Carter HB, Berndt SI, Ricker W, Schaeffer EM. Complications after prostate biopsy: data from SEER-Medicare. J Urol 2011;186:1830-4.

13. Loeb S, Vellekoop A, Ahmed HU, Catto J, Emberton M, Nam R, et al. Systematic review of complications of prostate biopsy. Eur Urol 2013;64:876-92.

14. Roberts MJ, Bennett HY, Harris PN, Holmes M, Grummet J, Naber K, et al. Prostate biopsy-related infection: a systematic review of risk factors, prevention strategies, and management approaches. Urology 2017;104:11-21.

15. Kasivisvanathan V, Rannikko AS, Borghi M, Panebianco V,
Mynderse LA, Vaarala MH, et al. MRI-targeted or standard biopsy for prostate-cancer diagnosis. N Engl J Med 2018; 378:1767-77.

16. Venderink $W$, van der Leest $M$, van Luijtelaar $A$, van de Ven WJM, Futterer JJ, Sedelaar JPM, et al. Retrospective comparison of direct in-bore magnetic resonance imaging (MRI)-guided biopsy and fusion-guided biopsy in patients with MRI lesions which are likely or highly likely to be clinically significant prostate cancer. World J Urol 2017;35: 1849-55.

17. Meier-Schroers M, Kukuk G, Wolter K, Decker G, Fischer S, Marx C, et al. Differentiation of prostatitis and prostate cancer using the Prostate Imaging-Reporting and Data System (PI-RADS). Eur J Radiol 2016;85:1304-11.

18. Jyoti R, Jina NH, Haxhimolla HZ. In-gantry MRI guided prostate biopsy diagnosis of prostatitis and its relationship with PIRADS V.2 based score. J Med Imaging Radiat Oncol 2017;61:212-5.

19. Panebianco V, Giganti F, Kitzing YX, Cornud F, Campa R, De Rubeis $\mathrm{G}$, et al. An update of pitfalls in prostate $\mathrm{mpM}$ RI: a practical approach through the lens of PI-RADS v. 2 guidelines. Insights Imaging 2018;9:87-101.

20. Wegelin O, van Melick HHE, Hooft L, Bosch JLHR, Reitsma HB, Barentsz JO, et al. Comparing three different techniques for magnetic resonance imaging-targeted prostate biopsies: a systematic review of in-bore versus magnetic resonance imaging-transrectal ultrasound fusion versus cognitive registration: is there a preferred technique? Eur Urol 2017;71:517-31.

21. Djavan B, Susani M, Bursa B, Basharkhah A, Simak R, Marberger M. Predictability and significance of multifocal prostate cancer in the radical prostatectomy specimen. Tech Urol 1999;5:139-42.

22. Andreoiu M, Cheng L. Multifocal prostate cancer: biologic, prognostic, and therapeutic implications. Hum Pathol 2010;41:781-93.

23. Wise AM, Stamey TA, McNeal JE, Clayton JL. Morphologic and clinical significance of multifocal prostate cancers in radical prostatectomy specimens. Urology 2002;60:264-9. 